\title{
Cumprimento às precauções-padrão por profissionais de enfermagem e fatores associados
}

\author{
Compliance with standard precautions by nursing professionals and related factors \\ Observancia de precauciones estándar por parte de profesionales de enfermería y factores asociados
}

\author{
Vitória Helena Pereira ${ }^{1}$ (1) \\ Leticia Nunes Torres² \\ Nathália Montanher Rodrigues ${ }^{1}$ (1) \\ Damiana Aparecida Trindade Monteiro ${ }^{3}$ (B) \\ Juliano Teixeira Moraes ${ }^{4}$ (1) \\ Fernanda Maria Vieira Pereira-Ávila ${ }^{5}$ (1) \\ Mariana Alvina dos Santos ${ }^{6}$ (C) \\ Elucir $\mathrm{Gir}^{7}$
}

Silmara Elaine Malaguti-Toffano ${ }^{1}$ (c)

1. Universidade Federal do Triângulo Mineiro. Uberaba, MG, Brasil.

2. Universidade Federal do Triângulo Mineiro Federal, Curso de Graduação em Enfermagem. Uberaba, MG, Brasil.

3. Universidade Federal do Triângulo Mineiro, Programa de Pós-Graduação em Atenção à Saúde. Uberaba, MG, Brasil.

4. Universidade Federal de São João del-Rei. São João del-Rei, MG, Brasil.

5. Universidade Federal Fluminense. Rio de Janeiro, RJ, Brasil.

6. Universidade Federal do Mato Grosso do Sul. Campo Grande, MS, Brasil.

7. Universidade de São Paulo, Escola de Enfermagem de Ribeirão Preto. Ribeirão Preto, SP, Brasil.

Autor Correspondente:

Silmara Elaine Malaguti-Toffano.

E-mail: silmalaguti@yahoo.com.br.

Recebido em 21/07/2020.

Aprovado em 09/12/2020.

DOl:https://doi.org/10.1590/2177-9465-EAN-2020-0193

\section{RESUMO}

Objetivo: Verificar o cumprimento às Precauções-Padrão por profissionais de enfermagem e fatores associados. Método: Estudo descritivo, transversal, com 522 profissionais de enfermagem, realizado em dois hospitais brasileiros, entre janeiro de 2017 a março de 2018. Os dados foram coletados por meio de um formulário contendo variáveis demográficas e profissionais e a Compliance with Standard Precautions Scale (versão Português-Brasil); posteriormente, analisados por estatísticas descritivas e exploratórias e um modelo de regressão linear múltiplo. Resultados: O escore médio global 12,9 (DP=2,5). Técnicos de enfermagem tiveram escores médios estatisticamente significativos maiores $(p<0,01)$ do que enfermeiros. Houve diferença significativa quanto à categoria profissional $(p<0,01)$ e escolaridade $(p<0,01)$, e, marginalmente significativa em relação à idade $(p=0,06)$. Não houve diferenças quanto à experiência profissional $(p=077)$, participação em treinamentos $(p=0,79)$, tipo de hospital $(p=0,13)$, respectivamente. A escolaridade não contribuiu para um maior cumprimento às medidas $(p<0,01)$, assim como o ensino superior $(p \leq 0,01)$. Conclusão e Implicações para a prática: $O$ aumento na escolaridade e experiência profissional não contribuiu para maior cumprimento às Precações. Por contemplar aspectos da prática de enfermagem, estratégias de prevenção de exposição ocupacional podem ser revistas e aperfeiçoadas.

Palavras-chave: Controle de Infecção; Precauções Universais; Equipe de Enfermagem; Exposição Ocupacional; Doenças Transmissíveis.

\section{AвstRAct}

Objective: To verify compliance with the Standard Precautions by nursing professionals and associated factors. Method: A descriptive, cross-sectional study was carried out with 522 nursing professionals, in two Brazilian hospitals, between January 2017 and March 2018. Data were collected using a form containing demographic and professional variables and the Compliance with Standard Precautions Scale (Portuguese-Brazilian version); later, analyzed by descriptive and exploratory statistics and a multiple linear regression model. Results: The global mean score was $12.9(\mathrm{SD}=2.5)$. Nursing professionals had statistically significant higher scores $(p<0.01)$ than nurses. There was a significant difference in terms of professional category $(p<0.01)$ ) and education $(p<0.01)$, and marginally significant in relation to age $(p=0.06)$. There were no differences regarding professional experience $(p=077)$, participation in training $(p=0.79)$, and type of hospital $(p=0.13)$, respectively. Education did not contribute to greater compliance with the measures $(p<0.01)$, nor did higher education $(p \leq 0.01)$. Conclusion and implications for practice: Increased education and professional experience did not contribute to greater compliance with the Standards Precautions. By considering aspects of nursing practice, occupational exposure prevention strategies can be reviewed and improved.

Keywords: Infection control; Universal Precautions; Nursing, Team; Occupational Exposure; Communicable Diseases.

\section{RESUMEN}

Objetivo: Verificar la observancia de las Precauciones Estándar por parte de profesionales de enfermería, y sus factores asociados. Método: Estudio descriptivo, transversal, con 522 profesionales de enfermería, realizado en dos hospitales brasileños entre enero de 2017 y marzo de 2018. Datos recolectados mediante formulario incluyendo variables sociodemográficas y profesionales, y Compliance with Standard Precautions Scale (versión Portugués-Brasil); analizados por estadística descriptiva y exploratoria y un modelo de regresión lineal múltiple. Resultados: Puntaje medio global de 12,9 (DS=2,5). Los auxiliares de enfermería obtuvieron puntajes promedio mayores, estadísticamente significantes $(p<0,01)$ respecto de los enfermeros. Existió diferencia significante respecto de la categoría profesional $(p<0,01)$ y la escolarización $(p<0,01)$; y marginalmente significante en relación a la edad $(p=0,06)$. No hubo diferencias relativas a la experiencia profesional $(p=0,77)$, participación en capacitaciones $(p=0,79)$ y tipos de hospital $(p=0,13)$. La escolarización no contribuyó a una mayor observancia de las medidas $(p<0,01)$, al igual que los estudios superiores $(p \leq 0,01)$. Conclusión e Implicaciones para la práctica: Mayores grados de escolarización y experiencia profesional no contribuyeron a la observancia de las Precauciones. En razón de contemplar aspectos de la práctica de enfermería, las estrategias de prevención y exposición profesional merecen ser revisadas y perfeccionadas.

Palabras clave: Control de Infecciones; Precauciones Universales; Grupo de Enfermería; Exposición Profesional; Enfermedades Transmisibles. 


\section{INTRODUÇÃO}

Profissionais da enfermagem estão vulneráveis aos riscos de exposição ocupacional, e, em decorrência do contato com sangue e outros fluídos corporais, estão expostos aos vírus da imunodeficiência humana (HIV), hepatite B (VHB) e C (VHC), conforme documentado na literatura. ${ }^{1}$

Considerando a pandemia da Covid-19 e a sobrecarga do sistema e do trabalho em saúde, a adoção de medidas específicas de proteção para os profissionais de saúde tornase um grande desafio. Além das altas taxas de infecção, os profissionais infectados podem ainda se tornar um veículo de transmissão do vírus para pacientes e outras pessoas com as quais mantêm contato, caso não adotem as medidas protetivas adequadamente. ${ }^{2,3}$

Considerando a pandemia da Covid-19 e a sobrecarga do sistema e do trabalho em saúde, a adoção às medidas específicas de proteção para os profissionais de saúde, se torna um grande desafio, pois além das altas taxas de infecção, os profissionais infectados podem ainda, se tornar um veículo de transmissão do vírus para pacientes e outras pessoas nas quais mantem contato, caso não adotem as medidas de proteção adequadamente. ${ }^{2,3}$

Com o intuito de proteger os profissionais da área da saúde, um conjunto de medidas, denominada Precauções-Padrão (PP), antigamente denominada Precauções-Universais foram recomendadas para todos os serviços de saúde, mediante possibilidade de contato com fluídos corporais, independente de diagnóstico presumido ou suspeito do paciente. ${ }^{4}$

As PP contemplam práticas essenciais para a prevenção e controle de infecção na assistência à saúde, como a higiene das mãos, o uso de luvas e a manutenção de um ambiente seguro, como o descarte correto dos materiais perfurantes ou cortantes e, ainda, a limpeza do chão e outras superfícies após derramamento de sangue..$^{2-5}$

Deste modo, por abranger medidas que contribuem para reduzir as infecções relacionadas à assistência à saúde (IRAS), como a higiene das mãos, os profissionais da saúde; saúde ao cumprirem as PP, promove também segurança aos pacientes sob seus cuidados. ${ }^{6-9}$

$\mathrm{O}$ cumprimento às PP pode ser influenciado por fatores individuais, psicossociais e organizacionais. ${ }^{5,7-11}$ Com o intuito de mensurar a adesão dos profissionais, pesquisas apontaram que, mesmo após mais de vinte anos de publicação das PP, os profissionais e estudantes da área de saúde, incluindo os da enfermagem, ainda têm dificuldades quanto ao cumprimento às recomendações. , $^{5,10-18}$

A baixa adesão à higiene das mãos, o uso incorreto, o desconhecimento e a desvalorização quanto ao uso de equipamentos de proteção individual (EPI), foram apontados como motivos de não cumprimento às PP. ${ }^{10,11,17}$

Considerando todos estes aspectos, este estudo teve como objetivo verificar o cumprimento às $\mathrm{PP}$ por profissionais de enfermagem, enfermagem, bem como os fatores associados.

\section{MÉTODO}

Trata-se de um estudo do tipo descritivo, transversal e quantitativo, realizado em dois hospitais brasileiros, sendo um público e o outro filantrópico, entre março de 2017 a janeiro de 2018.

O hospital público e de ensino é uma referência para 27 munícipios do Triângulo Mineiro para o atendimento de média e alta complexidade e contava com 320 leitos. Por sua vez, o hospital filantrópico, possuía 300 leitos e estava localizado na região Centro-Oeste de Minas Gerais, sendo referência para 53 municípios.

A população foi composta por 1240 profissionais de enfermagem, que atuavam em setores de internação (enfermarias) e de alta complexidade e que atendiam aos seguintes critérios de inclusão: exercer a função de enfermeiro, técnico e auxiliar de enfermagem; atuar na assistência direta e nos setores selecionados. Foram excluídos aqueles que no momento da coleta de dados exerciam cargos de chefia ou atividades administrativas.

Inicialmente, o calculo do tamanho amostral considerou uma proporção referente às categorias e instituições às quais os participantes pertenciam e ainda, um coeficiente de determinação $R^{2}=0,02$ em um modelo de regressão linear múltipla com 06 variáveis (nível de significância ou erro tipo 01 de $a=0,05$ e erro tipo II de $\beta=0,2$ ) e poder estatístico de $80 \%$. Utilizando o aplicativo Power Analysis and Sample Size (PAS), versão 13, introduzindo-se os valores supracitados, obteve-se um tamanho amostral de 674. Considerando-se uma perda de 15\% (recusas em participar), 775 participantes foram sorteados; no entanto, participaram 522 profissionais. A análise do poder a posteriori de um modelo de regressão linear múltipla, com seis variáveis e um coeficiente de determinação de $R^{2}=0,055$, indicou um poder estatístico de 99,1\%. Deste modo, as perdas não comprometeram o poder estatístico estipulado preliminarmente.

Os profissionais sorteados foram abordados e convidados pelos pesquisadores a participarem da pesquisa, em seu próprio turno de trabalho.

Foi aplicado um formulário, desenvolvido pelos pesquisadores, com variáveis demográficas e profissionais: sexo (feminino ou masculino), idade (em anos), categoria profissional (enfermeiros, técnicos de enfermagem ou auxiliares de enfermagem), tempo de experiência de trabalho (em anos), instituição (pública ou filantrópica), carga horária semanal (em horas), setor de Trabalho (alta complexidade ou enfermarias) e participação em Treinamento sobre Precauções-Padrão. Também foi aplicada a versão em Português Brasil da escala "Compliance with Standard Precautions Scale" (CSPS-PB).${ }^{15}$ Os instrumentos respondidos foram colocados pelo próprio participante em um envelope sem identificação, lacrado e entregue ao pesquisador ao término do preenchimento. Não foi permitida a entrega do instrumento em outro momento.

A CSPS é uma escala construída e validada para profissionais da enfermagem, do tipo Likert, com quatro opções de respostas (nunca, raramente, às vezes e sempre) que indicam a frequência do cumprimento às PP e composta por 20 itens, alocados em cinco dimensões. $\mathrm{O}$ instrumento foi considerado adequado 
para mensurar o cumprimento às PP e os autores da CSPSPB permitiram a aplicação e desenvolvimento deste estudo. ${ }^{15}$

Tendo em vista que os profissionais devem aderir integralmente às PP, era esperado que a opção "sempre" totalizasse a maioria das respostas. Em contrapartida, a opção "nunca" foi esperada aos itens invertidos (item 2, 4, 6 e 15); o escore individual da CSPS-PB varia de 0 a 20 e deste modo, quanto maior a pontuação, melhor o cumprimento às PP (média entre os grupos e percentual).

Os dados foram inseridos em uma planilha eletrônica do programa $E x c e / \AA$ para Windows $X P \AA$, validados por dupla entrada (digitação) e transferidos e analisados para o Statistical Package for the Social Sciences (SPSS), versão 19.0.

O cumprimento às PP (variável dependente) foi analisado segundo a pontuação máxima (20) no escore global da CSPS-PB. Para caracterizar os participantes e as variáveis demográficas e profissionais, medidas de tendência central (média e mediana) e de dispersão (desvio-padrão) foram usadas para avaliar as variáveis quantitativas; as categóricas, por sua vez, frequências absolutas e relativas. Para verificar se houve correlação entre a variável dependente e as variáveis independentes categóricas dicotomizadas (sexo, hospital de trabalho e participação em treinamentos sobre PP), o teste qui quadrado foi usado para avaliar para as variáveis nominais e para as ordinais, o teste de Spearman.

Com as variáveis independentes selecionadas segundo a literatura nacional e internacional pertinente para o desfecho, e, os coeficientes de regressão estimados, as variáveis independentes se mostraram satisfatórias ao modelo, quanto aos aspectos da linearidade, variância constante, independência e normalidade. Deste modo, categoria profissional, idade, escolaridade, hospital de trabalho, experiência profissional e participação em treinamento sobre PP foram indicadas para o modelo de regressão saturado (Método Enter).

Este estudo foi realizado em consonância com a resolução 466/12 do Conselho Nacional de Saúde. O projeto foi submetido à apreciação e aprovado pelo Comitê de Ética em Pesquisa (Parecer n.1.953.499/2017, CAAE: 457346616.1.0000.5154).

\section{RESULTADOS}

A maioria dos participantes era do sexo feminino ( $n=522 /$ $80,8 \%$ ), com idade média de 37,2 (DP $\pm 8,9$ ) anos e na função de técnico de enfermagem $(n=367 / 70,3 \%)$. As respostas apontaram mais de 10 anos de educação formal $(73,8 \%)$ e com atuação em enfermarias de cuidados clínicos ou cirúrgicos, em ambos os hospitais.

Com relação ao número de locais de trabalho, a maioria trabalhava em apenas um local $(90,8 \%)$, há menos de cinco anos (62,3\%) e com uma média de 42,1 horas (DP $\pm 12,0$ ) trabalhadas na semana na enfermagem, sendo o mínimo de 12 horas e máximo, 128. (Tabela 1 ).

Considerando o percentual de respostas "sempre", equivalente a $100 \%$ de cumprimento, para cada item da escala, a maioria dos participantes não teve resultados positivos quanto

Tabela 1. Distribuição e percentual de cumprimento às Precauções-Padrão por profissionais de enfermagem ( $n=522$ ), segundo itens da escala CSPS- PB. Uberaba-MG, 2017-2018.

\begin{tabular}{|c|c|c|c|c|c|c|c|c|}
\hline \multirow{3}{*}{ Itens da Escala de Cumprimento às Precauções-Padrão } & \multicolumn{4}{|c|}{ Enfermeiro } & \multicolumn{4}{|c|}{ Técnicos de Enfermagem } \\
\hline & \multicolumn{2}{|c|}{ Sim } & \multicolumn{2}{|c|}{ Não } & \multicolumn{2}{|c|}{ Sim } & \multicolumn{2}{|c|}{ Não } \\
\hline & $\mathrm{n}$ & $\%$ & $\mathrm{n}$ & $\%$ & $\mathrm{n}$ & $\%$ & $\mathrm{n}$ & $\%$ \\
\hline 1. Eu lavo minhas mãos entre contatos com pacientes. & 155 & 100 & - & - & 367 & 100 & - & - \\
\hline 2. Eu uso somente água para lavar as mãos. & 141 & 91,0 & 14 & 9,0 & 325 & 88,6 & 42 & 11,4 \\
\hline $\begin{array}{l}\text { 3. Eu uso produto à base de álcool para higienizar as mãos, } \\
\text { como alternativa, se não estiverem visivelmente sujas. }\end{array}$ & - & - & 155 & 100 & - & - & 367 & 100 \\
\hline 4. Eu reencapo agulhas usadas após aplicar uma injeção. & 149 & 96,1 & 06 & 3,9 & 351 & 95,6 & 16 & 4,4 \\
\hline $\begin{array}{l}\text { 5. Eu descarto materiais perfurocortantes em caixas } \\
\text { próprias. }\end{array}$ & - & - & 155 & 100 & - & - & 367 & 100 \\
\hline $\begin{array}{l}\text { 6. A caixa de materiais perfurocortantes é descartada } \\
\text { somente quando está cheia. }\end{array}$ & 89 & 57,4 & 66 & 42,6 & 193 & 52,6 & 174 & 47,4 \\
\hline $\begin{array}{l}\text { 7. Eu retiro os Equipamentos de Proteção Individual (EPI) em } \\
\text { um local designado. }\end{array}$ & - & - & 155 & 100 & - & - & 367 & 100 \\
\hline $\begin{array}{l}\text { 8. Eu tomo banho em caso de respingos extensos mesmo } \\
\text { que eu tenha usado EPI. }\end{array}$ & - & - & 155 & 100 & - & - & 367 & 100 \\
\hline $\begin{array}{l}\text { 9. Eu cubro meus ferimentos ou lesões com curativos à } \\
\text { prova d'água antes do contato com pacientes. }\end{array}$ & - & - & 155 & 100 & - & - & 367 & 100 \\
\hline $\begin{array}{l}\text { 10. Eu uso luvas quando estou exposto a fluidos corporais, } \\
\text { sangue ou derivados e qualquer excreção de pacientes. }\end{array}$ & - & - & 155 & 100 & - & - & 367 & 100 \\
\hline
\end{tabular}


Tabela 1. Continuação...

\begin{tabular}{|c|c|c|c|c|c|c|c|c|}
\hline \multirow{3}{*}{ Itens da Escala de Cumprimento às Precauções-Padrão } & \multicolumn{4}{|c|}{ Enfermeiro } & \multicolumn{4}{|c|}{ Técnicos de Enfermagem } \\
\hline & \multicolumn{2}{|c|}{ Sim } & \multicolumn{2}{|c|}{ Não } & \multicolumn{2}{|c|}{ Sim } & \multicolumn{2}{|c|}{ Não } \\
\hline & $\mathrm{n}$ & $\%$ & $\mathrm{n}$ & $\%$ & $\mathrm{n}$ & $\%$ & $\mathrm{n}$ & $\%$ \\
\hline 11. Eu troco de luvas entre contato com pacientes. & - & - & 155 & 100 & - & - & 367 & 100 \\
\hline $\begin{array}{l}\text { 12. Eu higienizo as minhas mãos imediatamente após } \\
\text { remover as luvas. }\end{array}$ & - & - & 155 & 100 & - & - & 367 & 100 \\
\hline $\begin{array}{l}\text { 13. Eu uso máscara cirúrgica ou em combinação com óculos } \\
\text { de proteção e avental sempre que houver a possibilidade de } \\
\text { respingos ou derramamentos. }\end{array}$ & - & - & 155 & 100 & - & - & 367 & 100 \\
\hline $\begin{array}{l}\text { 14. Minha boca e meu nariz ficam cobertos quando uso } \\
\text { máscara. }\end{array}$ & - & - & 155 & 100 & - & - & 367 & 100 \\
\hline 15. Eu reutilizo uma máscara cirúrgica ou EPI descartável. & 138 & 89,0 & 17 & 11,0 & 318 & 86,6 & 49 & 13,4 \\
\hline $\begin{array}{l}\text { 16. Eu uso avental/capote quando estou exposto a sangue, } \\
\text { fluídos corporais ou qualquer excreção de pacientes. }\end{array}$ & - & - & 155 & 100 & - & - & 367 & 100 \\
\hline $\begin{array}{l}\text { 17. Eu descarto material contaminado com sangue, fluidos } \\
\text { corporais, secreções e excreções de pacientes em sacos } \\
\text { plásticos brancos independentemente do estado infeccioso } \\
\text { do paciente. }\end{array}$ & - & - & 155 & 100 & - & - & 367 & 100 \\
\hline 18. Eu descontamino superfícies e equipamentos após o uso. & - & - & 155 & 100 & - & - & 367 & 100 \\
\hline $\begin{array}{l}\text { 19. Eu uso luvas para descontaminar equipamentos que } \\
\text { apresentam sujeira visível. }\end{array}$ & - & - & 155 & 100 & - & - & 367 & 100 \\
\hline $\begin{array}{l}\text { 20. Eu limpo imediatamente com desinfetante (álcool) } \\
\text { superfícies após derramamento de sangue ou outros fluidos } \\
\text { corporais. }\end{array}$ & - & - & 155 & 100 & - & - & 367 & 100 \\
\hline
\end{tabular}

ao cumprimento às $\mathrm{PP}$, principalmente, com relação ao uso de máscaras e óculos de proteção. A reutilização de máscaras foi apontada pela maioria dos participantes.

Os itens que tiveram um percentual maior de cumprimento por ambas as categorias estavam relacionados à higiene das mãos e reencape de agulhas contaminadas (Tabela 1).

A média do escore global de cumprimento às $P P(n=522)$ foi $12,8(\mathrm{DP} \pm 2,4)$, sendo o mínimo cinco, e máximo, 20. A média dos escores de cumprimento às PP foi maior entre o sexo masculino (Tabela 1).

Técnicos de enfermagem tiveram escores médios mais altos do que enfermeiros (Tabela 2) e os resultados apontaram uma correlação estatisticamente significativa entre os grupos $(p<0,01)$.

Os coeficientes de regressão estimados, as variáveis independentes se mostraram satisfatórias ao modelo, quanto aos aspectos da linearidade, variância constante, independência e normalidade. A análise resultou em um modelo estatisticamente significativo $\left[F_{(6,479)}=5,513 ; p<0,01 ; R^{2} 0,065\right]$, descrito na Tabela 3 .

Foram associados ao cumprimento das Precauções-Padrão os seguintes fatores: ser técnico de enfermagem $(p<0,01)$ e de maior escolaridade $(p<0,01)$, independente de idade $(p=0,06)$, experiência profissional $(p=077)$, participação em treinamentos $(p=0,79)$ e tipo de hospital $(p=0,13)$.

\section{DISCUSSÃO}

O presente estudo verificou o cumprimento às PP por profissionais de enfermagem e os fatores associados e os resultados apontaram que o cumprimento foi menor do que o esperado na maioria dos itens da escala. Categoria profissional e escolaridade foram os fatores associados ao cumprimento às PP.

A maioria dos participantes foi do sexo feminino, atuando como técnicas de enfermagem. No entanto, na análise dos escores médios de cumprimento às $\mathrm{PP}$, os homens apresentaram maior pontuação do que as mulheres. Estes dados corroboraram com outros estudos sobre cumprimento às PP que também teve maior participação de mulheres. ${ }^{13,15}$

Em relação à categoria profissional, houve maior cumprimento às PP entre os técnicos de enfermagem do que entre os enfermeiros, o que pode estar atrelado ao fato dos técnicos terem mais atividades relacionadas à realização de procedimentos que envolvem sangue e outros fluídos corporais do que os enfermeiros. Estes, como líderes, de equipe estão mais voltados 
Tabela 2. Associação dos escores médios de cumprimento às PP segundo grupos distintos de profissionais de enfermagem ( $N=522)$. Uberaba, 2017-2018

\begin{tabular}{|c|c|c|c|c|}
\hline Variável & $\mathrm{n}(\%)$ & Escores Médios & Desvio Padrão & $p$ \\
\hline \multicolumn{4}{|l|}{ Sexo } & \multirow{3}{*}{$0,19 *$} \\
\hline Feminino & $422(80,8)$ & 12,7 & 2,42 & \\
\hline Masculino & $100(19,2)$ & 13,1 & 2,63 & \\
\hline \multicolumn{4}{|l|}{ Categoria } & \multirow{3}{*}{$<0,01^{*}$} \\
\hline Enfermeiro & $155(29,6)$ & 12,3 & 2,53 & \\
\hline Técnicos de enfermagem & $367(70,3)$ & 13,0 & 2,39 & \\
\hline \multicolumn{4}{|l|}{ Hospital } & \multirow{3}{*}{$0,13^{*}$} \\
\hline Público & $399(76,4)$ & 12,9 & 2,51 & \\
\hline Filantrópico & $123(23,5)$ & 12,6 & 2,26 & \\
\hline \multicolumn{4}{|l|}{ Setor de Trabalho } & \multirow{3}{*}{$0,75^{*}$} \\
\hline Alta Complexidade & $288(55,1)$ & 12,8 & 2,48 & \\
\hline Enfermarias & $222(44,9)$ & 12,8 & 2,43 & \\
\hline \multicolumn{4}{|c|}{$\begin{array}{l}\text { Participação em Treinamento } \\
\text { sobre Precauções-Padrão }\end{array}$} & \multirow{3}{*}{$0,64 *$} \\
\hline Sim & $394(75,4 \%)$ & 12,8 & 2,48 & \\
\hline Não & $128(24,5 \%)$ & 12,9 & 2,41 & \\
\hline
\end{tabular}

* Teste Qui quadrado

Tabela 3. Regressão linear múltipla dos fatores relacionados ao cumprimento às Precauções Padrão por enfermeiros $(n=522)$. Uberaba, 2017- 2018

\begin{tabular}{|c|c|c|c|c|c|c|c|}
\hline \multirow[t]{2}{*}{ Variável } & \multirow[t]{2}{*}{ B } & \multirow{2}{*}{$\begin{array}{l}\text { Erro } \\
\text { Padrão }\end{array}$} & \multirow[t]{2}{*}{$\beta$} & \multirow[t]{2}{*}{$t$} & \multicolumn{2}{|c|}{$\begin{array}{c}\text { Intervalo de } \\
\text { Confiança (IC) 95\% } \\
\end{array}$} & \multirow[t]{2}{*}{$p$} \\
\hline & & & & & Mínimo & Máximo & \\
\hline Categoria Profissional &, 063 & ,241 & 0,11 & 0,26 & 0,14 & 1,03 & $\leq 0,01$ \\
\hline Idade* &, 590 & ,225 & 0,11 & 2,62 & $-0,00$ & 0,05 & 0,06 \\
\hline Escolaridade** &, 029 & ,016 & $-0,18$ & 1,83 & $-0,64$ & $-0,23$ & $\leq 0,01$ \\
\hline Hospital &,- 442 & 104 & $-0,01$ & $-4,23$ & $-0,55$ & 0,43 & 0,80 \\
\hline Experiência Profissional (em anos)*** &,- 005 & 018 & $-0,01$ & -0.24 & $-0,04$ & 0,03 & 0,77 \\
\hline Treinamento Precauções-Padrão &,- 062 & ,252 & 0,01 & $-0,28$ & $-0,41$ & 0,53 & 0.79 \\
\hline
\end{tabular}

${ }^{1}$ Variável dependente: Escore médio de cumprimento as Precauções-Padrão; *Dados ausentes 5.3\% $(n=494) ;{ }^{* *}$ Dados ausentes $0.1 \%$ ( $\left.n=521\right) ; * * *$ Dados ausentes $0.1 \%(n=521)$

para a supervisão destas atividades. Esses resultados diferem de outros estudos autorreferidos. $14,15,19$

A maioria dos técnicos de enfermagem relatou ter um bacharelado ou bacharelado em enfermagem ou em outra área. No entanto, de acordo com a análise de regressão e contrariamente à hipótese, o aumento dos anos de escolaridade não mostrou associação com o aumento do cumprimento às PP. Os resultados foram diferentes de outro estudo no qual o nível acadêmico de enfermagem foi um preditor associado ao aumento de cumprimento às PP. ${ }^{17}$

No Brasil, a equipe de enfermagem é composta por categorias distintas, cujas diferenças estão relacionadas aos anos de estudo.
Nesta pesquisa, houve a participação de enfermeiros, com quatro ou cinco anos de bacharelado e de técnicos de enfermagem, com dois anos de curso específico. Na prática clínica, estas duas categorias assumem responsabilidades distintas e os técnicos realizam mais procedimentos envolvendo fluidos corporais, o que pode justificar maiores escores de cumprimento às PP.

O tipo de hospital ou setor de trabalho não foi considerado como variáveis preditoras para o cumprimento às PP, embora tenham sido observadas diferenças nos escores médios destas variáveis. Os resultados apontaram que a maioria dos participantes desta investigação trabalhava somente em um único hospital. Outros estudos apontaram que boa parte dos profissionais de 
enfermagem mantem duplo vínculo de trabalho e com carga horária alta, ${ }^{20-22}$ o que pode acarretar aumento nos riscos de exposição a material biológico potencialmente contaminado e ainda, influenciar no cumprimento às PP.

A atuação em um único local de trabalho poderia ser explicada pela mudança de gestão do hospital público, cuja admissão da maioria dos profissionais ocorreu nos últimos cinco anos anteriores à coleta de dados da pesquisa. Tal situação também foi apontada em outro estudo realizado no mesmo estado, em um hospital público, com mesmo perfil. ${ }^{23}$

Estudos anteriores mostraram que a carga horária de trabalho semanal pode influenciar no cumprimento às PP. ${ }^{14,23-25}$ A carga de trabalho não foi avaliada no modelo de regressão do presente estudo, em virtude da maioria atuar em um único local.

De acordo com um estudo chinês, os gerentes de hospitais precisam aumentar os recursos humanos adequadamente, de modo a reduzir a intensidade do trabalho e ainda contribuir para melhorar o cumprimento às $\mathrm{PP}$, diminuindo também os riscos ocupacionais. ${ }^{14}$

Os resultados desta investigação podem contribuir para a discussão de aspectos relacionados aos programas de educação em enfermagem e mercado de trabalho. Considerando que nos últimos anos no Brasil houve um aumento nas vagas no ensino superior e de pós-graduação, o que contribuiu para um avanço na formação da enfermagem e para o aumento destes profissionais à beira do leito, ${ }^{20,21}$ esperava-se maior cumprimento às $\mathrm{PP}$, o que não foi apontado nos resultados, por não ser objeto desta investigação.

Não foi observada também uma diferença significativa no cumprimento às PP entre os profissionais de enfermagem que realizaram ou não treinamentos sobre as PP. Esse achado difere de outro estudo realizado na Itália, em que os participantes de, pelo menos, um treinamento exibiram escores de cumprimento mais altos do que os outros que não o fizeram. ${ }^{16}$

As diferenças de cumprimento às PP entre as categorias de enfermagem podem ser consideradas no planejamento de estratégias para prevenir exposições ocupacionais e infecções relacionadas à assistência à saúde.

Considerando a pandemia da Covid-19 autores de uma investigação apontaram que as PP são extremamente necessárias para a proteção dos profissionais de saúde e por isto, é importante que aprendam e valorizem medidas de prevenção desde a sua formação. Para tanto, há necessidade de revisão nos currículos de graduação, para que, no futuro, os profissionais possam estar preparados para o enfrentamento de doenças emergentes e que valorizem não só para as medidas individuais, mas principalmente a gestão dos EPI nos serviços de saúde. ${ }^{26}$

Levando em conta a amostra estudada, os resultados não podem ser extrapolados para todos os profissionais de enfermagem que atuam em hospitais, o que pode ser considerado como limite do estudo. Outro ponto está relacionado ao fato de o instrumento ter sido aplicado no próprio local de trabalho, além de ter sido respondido pelos próprios participantes, o que pode não refletir, em sua totalidade, a realidade do cumprimento às PP. Por fim, a reprodução desta pesquisa por meio eletrônico ou em ambiente domiciliar poderá trazer resultados distintos.

A CSPS-PB contempla aspectos da prática clínica da enfermagem e os resultados desta investigação podem contribuir para a realização de ações de educação em serviço e ainda, direcionar as estratégias de prevenção de exposição ocupacional envolvendo material biológico.

\section{CONCLUSÃO}

O cumprimento às PP foi menor que o esperado para a maioria dos itens da escala. Além disso, uma idade mais jovem foi associada a um menor cumprimento. Dentre as diferentes categorias profissionais, os enfermeiros apresentaram maiores escores do que os técnicos de enfermagem. O ensino superior contribuiu negativamente para o cumprimento às $\mathrm{PP}$. Os resultados foram diferentes dos esperados, uma vez que a maioria dos participantes tinha curso superior e recebeu treinamento sobre o cumprimento às PP.

\section{FINANCIAMENTO}

Fundação de Amparo à Pesquisa de Minas Gerais à pesquisa com o título "Cumprimento às Precauções-Padrão entre profissionais de enfermagem em hospitais de Minas Gerais" (processo número: APQ/00149-16), concedida à autora Silmara Elaine Malaguti Toffano.

\section{AGRADECIMENTOS}

Ao professor Dr. Vanderlei Hass pelas contribuições na análise estatística.

\section{CONTRIBUIÇÕES DOS AUTORES}

Desenho do estudo. Silmara Elaine Malaguti-Toffano.

Coleta ou produção dos dados. Damiana Aparecida Trindade Monteiro. Juliano Teixeira Moraes. Nathália Montanher Rodrigues. Leticia Nunes Torres. Vitória Helena Pereira.

Análise de dados. Silmara Elaine Malaguti-Toffano. Damiana Aparecida Trindade Monteiro. Nathália Montanher Rodrigues. Leticia Nunes Torres. Vitória Helena Pereira. Elucir Gir. Mariana Alvina dos Santos.

Interpretação dos resultados. Silmara Elaine Malaguti-Toffano. Damiana Aparecida Trindade Monteiro. Juliano Teixeira Moraes. Nathália Montanher Rodrigues. Leticia Nunes Torres. Vitória Helena Pereira. Elucir Gir. Mariana Alvina dos Santos.

Redação e revisão crítica do manuscrito. Vitória Helena Pereira. Leticia Nunes Torres. Nathália Montanher Rodrigues. Damiana Aparecida Trindade Monteiro. Juliano Teixeira Moraes. Fernanda Maria Vieira Pereira-Ávila. Mariana Alvina dos Santos. Elucir Gir. Silmara Elaine Malaguti-Toffano.

Aprovação da versão final do artigo. Vitória Helena Pereira. Leticia Nunes Torres. Nathália Montanher Rodrigues. Damiana Aparecida Trindade Monteiro. Juliano Teixeira Moraes. Fernanda 
Maria Vieira Pereira-Ávila. Mariana Alvina dos Santos. Elucir Gir. Silmara Elaine Malaguti-Toffano.

Responsabilidade por todos os aspectos do conteúdo e a integridade do artigo publicado. Vitória Helena Pereira. Leticia Nunes Torres. Nathália Montanher Rodrigues. Damiana Aparecida Trindade Monteiro. Juliano Teixeira Moraes. Fernanda Maria Vieira Pereira-Ávila. Mariana Alvina dos Santos. Elucir Gir. Silmara Elaine Malaguti-Toffano.

\section{EDITOR ASSOCIADO}

Rafael Celestino da Silva

\section{REFERÊNCIAS}

1. Motaarefi $\mathrm{H}$, Mahmoudi $\mathrm{H}$, Mohammadi E, Hasanpour-Dehkordi A. Factors associated with needlestick injuries in health care occupations: a systematic review. J Clin Diagn Res. 2016;10(8):IE01-04. http://dx.doi. org/10.7860/JCDR/2016/17973.8221. PMid:27656466.

2. Delgado D, Wyss-Quintana F, Perez G, Sosa-Liprandi A, Ponte-Negretti C, Mendoza I et al. Personal Safety during the COVID-19 pandemic: realities and perspectives of healthcare workers in Latin America. Int $J$ Environ Res Public Health. 2020;17(8):2798. https://doi.org/10.3390/ ijerph17082798.

3. Verbeek JH, Rajamaki B, ljaz S, Tikka C, Ruotsalainen JH, Edmond $\mathrm{MB}$ et al. Personal protective equipment for preventing highly infectious diseases due to exposure to contaminated body fluids in healthcare staff. Cochrane Database Syst Rev. 2019;7:CD011621. http://dx.doi. org/10.1002/14651858.CD011621.pub3.

4. Moralejo D, EI Dib R, Prata RA, Barretti P, Corrêa I. Improving adherence to Standard Precautions for the control of health care-associated infections. Cochrane Database Syst Rev. 2018;2:CD010768. https:// doi.org/10.1002/14651858.CD010768.pub2.

5. Lam SC. Validation and cross-cultural pilot testing of compliance with standard precautions scale: self-administered instrument for clinical nurses. Infect Control Hosp Epidemiol. 2014;35(5):547-55. http://dx.doi. org/10.1086/675835. PMid:24709724.

6. Martos-Cabrera MB, Mota-Romero E, Martos-García R, Gómez-Urquiza JL, Suleiman-Martos N, Albendín-García L et al. Hand hygiene teaching strategies among nursing staff: a systematic review. Int J Environ Res Public Health. 2019;16(17):3039. https://doi.org/10.3390/ijerph16173039.

7. Williams VR, Leis JA, Trbovich P, Agnihotri T, Lee W, Joseph B et al. Improving healthcare worker adherence to the use of transmissionbased precautions through application of human factors design: a prospective multi-centre study. J Hosp Infect. 2019;103(1):101-5. http:// dx.doi.org/10.1016/j.jhin.2019.03.014. PMid:30935983.

8. Zingg W, Holmes A, Dettenkofer M, Goetting T, Secci F, Clack L et al. Hospital organization, management, and structure for prevention of healthcare-associated infection: a systematic review and expert consensus. Lancet Infect Dis. 2015;15(2):212-24. http://dx.doi.org/10.1016/S14733099(14)70854-0. PMid:25467650.

9. Gould DJ, Moralejo D, Drey N, Chudleigh JH, Taljaard M. Interventions to improve hand hygiene compliance in patient care. Cochrane Database Syst Rev. 2017;9:CD005186. http://dx.doi.org/10.1002/14651858. CD005186.pub4. PMid:28862335.

10. Bouchoucha SL, Moore KA. Factors influencing adherence to standard precautions Scale: a psychometric validation. Nurs Health Sci. 2019 jun;21(2):178-85. http://dx.doi.org/10.1111/nhs.12578.

11. Powers D, Armellino D, Dolansky M, Fitzpatrick J. Factors influencing nurse compliance with Standard Precautions. Am J Infect Control. 2016;44(1):47. http://dx.doi.org/10.1016/j.ajic.2015.10.001. PMid:26769280.
12. Beyamo A, Dodicho T, Facha W. Compliance with standard precaution practices and associated factors among health care workers in Dawuro Zone, South West Ethiopia, cross sectional study. BMC Health Serv Res. 2019;19:381. https://doi.org/10.1186/s12913-019-4172-4.

13. Alshammari F, Cruz JP, Alquwez N, Almazan J, Alsolami F, Tork H et al Compliance with standard precautions during clinical training of nursing students in Saudi Arabia: a multi-university study. J Infect Dev Ctries. 2018;12(11):937-45. https://doi.org/10.3855/jidc.10821.

14. Quan $M$, Wang X, Wu H, Yuan X, Lei D, Jiang Z et al. Influencing factors on use of standard precautions against occupational exposures to blood and body fluids among nurses in China. Int $\mathrm{J}$ Clin Exp Med. 2015;8(12):22450-9. PMID: 26885227.

15. Pereira FMV, Lam SC, Gir E. Cultural adaptation and reliability of the Compliance with Standard Precautions Scale (CSPS) for nurses in Brazil. Rev Lat Am Enfermagem. 2017;25:e2850. http://dx.doi. org/10.1590/1518-8345.1204.2850. PMid:28301030.

16. Donati D, Biagioli V, Cianfrocca C, De Marinis MG, Tartaglini D. Compliance with standard precautions among clinical nurses: validity and reliability of the Italian version of the Compliance with Standard Precautions Scale (CSPS-It). Int J Environ Res Public Health. 2019;16(1):121. http://dx.doi. org/10.3390/ijerph16010121. PMid:30621210.

17. Haile TG, Engeda EH, Abdo AA. Compliance with standard precautions and associated factors among healthcare workers in Gondar University comprehensive specialized hospital, Northwest Ethiopia. J Environ Public Health. 2017;2017:2050635. http://dx.doi.org/10.1155/2017/2050635. PMid:28191020.

18. Morioka S, Tajima T, SugikiY, Hayakawa K, Ohmagari N. Adherence to personal protective equipment use among nurses in Japanese tertiary care hospitals: what determines variability? J Hosp Infect. 2020;104(3):344-9. http://dx.doi.org/10.1016/j.jhin.2019.11.019. PMid:31790746.

19. Maroldi MAC, Felix AMA, Dias AAL, Kawagoe JY, Padoveze MC, Ferreira $\mathrm{SA}$ et al. Adherence to precautions for preventing the transmission of microorganisms in primary health care: a qualitative study. BMC Nurs. 2017;16(1):49. http://dx.doi.org/10.1186/s12912-017-0245-z. PMid:28919838.

20. Frota MA, Wermelinger MCMW, Vieira LJES, Ximenes No FRG, Queiroz RSM, Amorim RF. Mapeando a formação do enfermeiro no Brasil: desafios para atuação em cenários complexos e globalizados. Cien Saude Colet. 2020 jan;25(1):25-35. http://dx.doi.org/10.1590/141381232020251.27672019. PMid:31859852.

21. Silva KL, de Sena RR, Tavares TS, Belga SM, Maas LW. Migrant nurses in Brazil: demographic characteristics, migration flow and relationship with the training process. Rev Lat Am Enfermagem. 2016;24(0):e2686. http://dx.doi.org/10.1590/1518-8345.0390.2686. PMid:27027681.

22. Silva RSS, Madeira MZA, Fernandes MA, Batista OMA, Brito BAM, Carvalho NAR. Riscos ocupacionais entre trabalhadores de enfermagem em Unidade de Terapia Intensiva. Rev Bras Med Trab. 2017;15(3):26775. http://dx.doi.org/10.5327/Z1679443520170027. PMid:32270067.

23. Carvalho PCF, Januário GC, Monteiro DAT, Toffano SEM. Conhecimento e atitudes da equipe de enfermagem sobre cateter com dispositivo seguro em um hospital brasileiro. Rev. Enferm. UFSM. 2019;9(e50):117. http://dx.doi.org/10.5902/2179769234767.

24. Lim JH, Ahn JW, Son YJ. Association between Hospital Nurses' perception of patient safety management and standard precaution adherence: a cross-sectional study. Int J Environ Res Public Health. 2019;16(23):4744 http://dx.doi.org/10.3390/ijerph16234744. PMid:31783559.

25. Cui Z, Zhu J, Zhang X, Wang B, Li X. Sharp injuries: a cross-sectional study among health care workers in a provincial teaching hospital in China. Environ Health Prev Med. 2018;23(2):1-7. http://dx.doi.org/10.1186/ s12199-017-0691-y.

26. Rose S. Medical student education in the time of COVID-19. JAMA 2020;323(21):2131-2. http://dx.doi.org/10.1001/jama.2020.5227. PMid:32232420. 